REVISTA DE LA

UNIÓN MATEMÁTICA ARGENTINA

Vol. 59, No. 2, 2018, Pages 431-441

Published online: July 10, 2018

\title{
GENERALIZATIONS OF HYPERBOLIC AREA FOR TOPOLOGICAL SURFACES
}

\author{
ALDO-HILARIO CRUZ-COTA
}

\begin{abstract}
We introduce two generalizations of hyperbolic area for connected, closed, orientable surfaces: the complexity and the simple complexity of a surface. These concepts are defined in terms of collections of branched coverings $M \rightarrow \mathbb{P}^{1}$, where $M$ is a Riemann surface homeomorphic to $S$ and $\mathbb{P}^{1}$ is the Riemann sphere. We prove that if $S$ is a surface of positive genus, then both the topological complexity and the simple topological complexity of $S$ are linear functions of its genus.
\end{abstract}

\section{INTRODUCTION}

In this article we study the simplest way in which a given Riemann surface can be realized as a branched cover of the Riemann sphere. The simplicity of a branched cover is defined in terms of its complexity, which is defined below.

Let $p$ be a branched covering of a Riemann surface $M$ to the Riemann sphere $\mathbb{P}^{1}$. Let $X$ be the complement of the branching set of $p$ in $\mathbb{P}^{1}$. Since $X$ is a domain of the Riemann sphere, then it naturally has the structure of a Riemann surface. We define the complexity of the branched covering $p$ as the product of its degree and the area of $X$. Here, the area of $X$ is defined as its hyperbolic area, if $X$ admits a hyperbolic structure, and infinity otherwise.

The above definition of complexity is a generalization of that introduced in [2] and is motivated as follows. Given a Riemann surface $M$, one can adopt the topological point of view that the simplicity of a branched covering $M \rightarrow \mathbb{P}^{1}$ is determined by its degree and the cardinality of its branching set (taking into account the multiplicities or degrees of the branching points). From a geometric point of view, however, a more natural invariant to consider is the area of the complement of the branching set in the Riemann sphere. This is in accordance with a principle usually adopted in three-dimensional topology: the hyperbolic volume somehow measures how complicated a hyperbolic manifold is. The definition of complexity given above encompasses both the topological and geometric points of view.

For a Riemann surface $M$, we define its complexity as the infimum of the set of all the complexities of branched coverings $M \rightarrow \mathbb{P}^{1}$. Thus, the complexity of a

2010 Mathematics Subject Classification. Primary 57M12; Secondary 30F99.

Key words and phrases. Branched coverings; Complexity of surfaces. 
Riemann surface $M$ measures the most economical way in which $M$ can be realized as a branched cover of the Riemann sphere $\mathbb{P}^{1}$.

We extend the definition of complexity to topological surfaces as follows. If $S$ is a surface, we define its topological complexity as the infimum of the set of all the complexities of the Riemann surfaces that are homeomorphic to $S$. The complexity of a surface measures how complicated the surface is. Because of that, we can think of the complexity for surfaces as a 2-dimensional topological analog of the hyperbolic volume for hyperbolic 3-manifolds. The definition of complexity also has the advantage that is defined for all surfaces.

In [2], the authors found a formula for the complexity of a Riemann surface, but that formula is in terms of an integer that is hard to find in practice, mainly because of its close connection to the Hurwitz problem for the sphere. This problem is very difficult: it has been open for more than a century and is still open today in its full generality (see the next paragraph). The raison d'être for this article was to find explicit formulas for the topological complexity of a surface that did not involve using the general Hurwitz problem. One of the main ingredients in the proofs of these formulas was adapting a variety of partial solutions to the Hurwitz problem to our context.

The Hurwitz problem for the sphere essentially asks when a collection of partitions of a positive integer can be realized as the combinatorial data of a branched covering of the sphere. This problem was first studied by Hurwitz in [7, albeit in the more general case in which the sphere is replaced by an arbitrary surface. Since then, many people have studied the Hurwitz problem, such as the authors of [3, 6, 8, 4, 1, 10, 11, 9]. In particular, we now know that the problem has been solved for almost all surfaces. The only instances of the problem that remain open reduce to the Hurwitz problem for the sphere (see [10, Section 2]).

There is another classical invariant for topological surfaces: the genus. Our first main theorem relates the complexity of a topological surface to its genus.

Main Theorem 1. Let $S$ be a connected, closed, orientable surface of genus $g$. Let $C_{\text {top }}(S)$ denote the topological complexity of $S$. Then

$$
C_{\mathrm{top}}(S)= \begin{cases}2 \pi(2 g+1) & \text { if } g \geq 1, \\ 6 \pi & \text { if } g=0 .\end{cases}
$$

We say that a branched covering $p: M \rightarrow \mathbb{P}^{1}$ of degree $d$ is simple if the cardinality of $p^{-1}(y)$ is at least $d-1$ for all $y \in \mathbb{P}^{1}$. We define the simple topological complexity of a surface $S$ as the infimum of the complexities of all simple branched coverings $M \rightarrow \mathbb{P}^{1}$ with $M$ homeomorphic to $S$. Our second main result gives us explicit formulas for the simple topological complexity of a surface.

Main Theorem 2. Let $S$ be a connected, closed, orientable surface of genus $g$. Let $C_{\text {simp }}(S)$ denote the simple topological complexity of $S$. Then

$$
C_{\text {simp }}(S)= \begin{cases}8 \pi g & \text { if } g \geq 1 \\ 12 \pi & \text { if } g=0\end{cases}
$$


Our proofs of the two main theorems are constructive; they exhibit the combinatorial data of branched coverings of minimal complexity (among those coverings whose domains have a fixed topological type).

Notation 1.1. Throughout this article, all Riemann and topological surfaces will be assumed to be connected, closed and orientable, unless otherwise stated. A Riemann surface will be denoted by $M$, except in the case of the Riemann sphere, which we denote by $\mathbb{P}^{1}$. A topological surface will be denoted by $S$. The topological 2 -sphere will be denoted by $\mathbb{S}^{2}$. We use $\chi(M)$ to denote the Euler characteristic of the surface $M$. The infimum of a subset $A$ of the extended real line $[-\infty, \infty]$ will be denoted by $\inf (A)$. A sequence of positive integers in square brackets represents the elements of a partition. For example, $[1,1,1]$ denotes the trivial partition $3=1+1+1$. The number of elements of a partition is called its length. For example, $[5,3]$ is a partition of 8 of length 2 . The cardinality of a set $B$ will be denoted by $|B|$.

\section{2. $(d, n)$-Branched COVERINGS AND the HuRWitz PROBlem}

Definition 2.1. A $(d, n)$-branched covering is a branched covering $p: M \rightarrow \mathbb{P}^{1}$ that satisfies the following conditions:

- $M$ is a Riemann surface.

- The degree of $p$ is $d$.

- The cardinality of the branching set of $p$ is $n$.

For $(d, n)$-branched coverings, the Riemann-Hurwitz formula takes the following form.

Theorem 2.2. Let $p: M \rightarrow \mathbb{P}^{1}$ be $a(d, n)$-branched covering with branching set $B$. If the pre-image $p^{-1}(B)$ has cardinality $m$, then

$$
\chi(M)-m=d\left(\chi\left(\mathbb{P}^{1}\right)-n\right) .
$$

Let $p: M \rightarrow \mathbb{P}^{1}$ be a $(d, n)$-branched covering. Suppose that $y_{1}, y_{2}, \ldots, y_{n}$ are all the distinct branch points of the branched covering. For each $i=1,2, \ldots, n$, the ramification indices of the points in $p^{-1}\left(y_{i}\right)$ form a partition $\Pi_{i}$ of the integer $d$. We gather these partitions in a single collection $\Pi=\left\{\Pi_{1}, \Pi_{2}, \ldots, \Pi_{n}\right\}$ (with repetitions allowed). We call the triplet $(d, n, \Pi)$ the branch triplet of the branched covering $p: M \rightarrow \mathbb{P}^{1}$.

We generalize the idea of branch triplet as follows.

Definition 2.3. The triplet $(d, n, \Pi)$ is called an abstract branch triplet if the following conditions are satisfied:

- $d$ and $n$ are positive integers;

- $\Pi$ is a collection (with repetitions allowed) of $n$ partitions of $d$, none of which is the trivial partition $[1,1, \ldots, 1]$ of $d$.

The sum of the lengths of the partitions in $\Pi$ is called the total length of the abstract branch triplet. 
Definition 2.4. Let $\mathcal{T}$ be an abstract branch triplet and let $M$ be a Riemann surface. We say that $\mathcal{T}$ is realizable on $M$ if there exists a branched covering $p: M \rightarrow \mathbb{P}^{1}$ whose branch triplet is equal to $\mathcal{T}$. An abstract branch triplet is called realizable if it is realizable on a Riemann surface.

A natural question to ask is the following:

Question 1. What abstract branch triplets are realizable?

The Riemann-Hurwitz formula gives a necessary condition for an abstract branch triplet to be realizable. For if $(d, n, \Pi)$ is an abstract branch triplet of total length $m$ that is realizable on a Riemann surface $M$, then:

$$
\chi(M)-m=d(2-n) .
$$

Definition 2.5. An abstract branch triplet $(d, n, \Pi)$ of total length $m$ that satisfies equation 2.1) is called compatible with $M$.

Every abstract branch triplet that is realizable on a Riemann surface $M$ is compatible with $M$. However, the converse of the last statement is not true (see [3. Corollary 6.4]). The problem of determining what compatible branch triplets are realizable as branched coverings of the sphere is known as the Hurwitz problem for the sphere.

In the rest of the section we collect the partial solutions to the Hurwitz problem that we need to prove our main theorems. We start with a classical result of Edmonds, Kulkarni and Stong ( $[3]$ ), who studied the problem of realizability of topological branched covers, mainly in the algebraic setting of representations of fundamental groups of punctured surfaces into symmetric groups.

Theorem 2.6 ([3, Proposition 5.2]). Let $\mathcal{T}=(d, n, \Pi)$ be an abstract branch triplet of total length $m$. Suppose that the length of one of the partitions in $\Pi$ is one, and that $n d-m$ is an even number such that $n d-m \geq 2 d-2$. Then $\mathcal{T}$ is realizable.

The next two results are corollaries of Theorem 2.6

Lemma 2.7. The abstract branch triplet $\mathcal{T}=(3,3,\{[3],[1,2],[1,2]\})$ is realizable on a Riemann surface homeomorphic to the sphere.

Theorem 2.8. Let $S$ be a surface of genus $g \geq 1$ and let $d=2 g+1$. Then the abstract branch triplet $\mathcal{T}=(d, 3,\{[d],[d],[d]\})$ is realizable on a Riemann surface that is homeomorphic to $S$.

In [1, Barański approached the Hurwitz problem in the setting of rational maps on the Riemann sphere. Using his geometric criterion ([1, Lemma 5]), Barański proved the following.

Theorem 2.9 ([1, Theorem 12]). Let $\mathcal{T}=(d, n, \Pi)$ be an abstract branch triplet with $n \geq d$. Suppose that $\mathcal{T}$ is compatible with the topological 2 -sphere $\mathbb{S}^{2}$. Then $\mathcal{T}$ is realizable by a Riemann surface homeomorphic to $\mathbb{S}^{2}$.

Theorems 2.6 and 2.9 are also mentioned in [10, Section 2], where Pervova and Petronio compile an extensive list of known results and techniques related to the Hurwitz problem. 


\section{DifFERENT NOTIONS OF COMPLEXITY}

We define several notions of complexity: one for branched coverings, another for Riemann surfaces, and the last one for topological surfaces.

Definition 3.1. Let $p: M \rightarrow \mathbb{P}^{1}$ be a branched covering of degree $d$ of the Riemann surface $M$ to the Riemann sphere $\mathbb{P}^{1}$. Let $B \subset \mathbb{P}^{1}$ be the branching set of $p$. Then the complement $\mathbb{P}^{1} \backslash B$ of $B$ in $\mathbb{P}^{1}$ inherits the structure of a Riemann surface from the Riemann sphere $\mathbb{P}^{1}$.

(1) We call the branched covering $p: M \rightarrow \mathbb{P}^{1}$ hyperbolic if $\mathbb{P}^{1} \backslash B$ admits a hyperbolic structure. In this case, we denote the hyperbolic area of $\mathbb{P}^{1} \backslash B$ by $\mathcal{A}\left(\mathbb{P}^{1} \backslash B\right)$.

(2) We define the complexity $C_{\mathrm{Cov}}(p)$ of the branched covering $p$ as follows:

$$
C_{\mathrm{Cov}}(p)= \begin{cases}d \cdot \mathcal{A}\left(\mathbb{P}^{1} \backslash B\right) & \text { if } p \text { is hyperbolic, } \\ \infty & \text { otherwise. }\end{cases}
$$

Remark 3.2. By [5, Theorem 27.12], a $(d, n)$-branched covering is hyperbolic if and only if $n \geq 3$.

The complexity of a hyperbolic branched covering is a mixture of a topological invariant, the degree, and a geometric invariant, the hyperbolic area of the complement of the branching set in $\mathbb{P}^{1}$. Thus, we can use the Gauss-Bonnet theorem to find an explicit formula for the complexity of a hyperbolic branched covering.

\section{Lemma 3.3.}

(1) The hyperbolic area of the complement of $n \geq 3$ points in the Riemann sphere equals $2 \pi(n-2)$.

(2) The complexity of a $(d, n)$-branched covering with $n \geq 3$ is finite and equal to $2 \pi d(n-2)$.

Proof.

(1) Let $\mathcal{A}\left(M_{n}\right)$ denote the hyperbolic area of the complement $M_{n}$ of $n \geq 3$ points in the Riemann sphere $\mathbb{P}^{1}$. It follows from the Gauss-Bonnet theorem that $\mathcal{A}\left(M_{n}\right)=-2 \pi \chi\left(M_{n}\right)=-2 \pi\left(\chi\left(\mathbb{P}^{1}\right)-n\right)=2 \pi(n-2)$.

(2) Let $p: M \rightarrow \mathbb{P}^{1}$ be a $(d, n)$-branched covering with $n \geq 3$. Let $B$ be the branching set of the covering $p$. By Remark $3.2, \mathbb{P}^{1} \backslash B$ admits a hyperbolic structure. Thus, the complexity $C(p)$ of $p$ is equal to

$$
C(p)=d \cdot \mathcal{A}\left(\mathbb{P}^{1} \backslash B\right)=d \mathcal{A}\left(M_{n}\right)=2 \pi d(n-2) .
$$

Combining Remark 3.2 and Lemma 3.3 we obtain the following.

Corollary 3.4. For a $(d, n)$-branched covering $p: M \rightarrow \mathbb{P}^{1}$, the following statements are equivalent:

(1) $p: M \rightarrow \mathbb{P}^{1}$ is hyperbolic;

(2) $n \geq 3$;

(3) $C_{\mathrm{Cov}}(p)<\infty$. 
Let $\mathscr{C}$ be the set of all complexities of branched coverings of Riemann surfaces to the Riemann sphere. Let $\mathbb{Z} \pi$ be the set of all integer multiples of the number $\pi$. Since $\mathscr{C}$ is a subset of $\mathbb{Z} \pi \cup\{\infty\}$ and $\mathbb{Z} \pi$ is a discrete subset of the real line, then we have the following:

Observation 3.5. The infimum of a non-empty subset of $\mathscr{C}$ is attained, although it could be infinite.

We now define the complexity of a Riemann surface. It essentially measures the most economical way in which the Riemann surface can be expressed as a branched covering of the Riemann sphere.

Definition 3.6. We define the complexity, $C_{\text {Riem }}(M)$, of a Riemann surface $M$ as the infimum of the set of all complexities of branched coverings of $M$ to the Riemann sphere:

$$
C_{\text {Riem }}(M)=\inf \left\{C_{\mathrm{Cov}}(p) \mid p: M \rightarrow \mathbb{P}^{1} \text { is a branched covering }\right\} .
$$

It is a well-known fact that every compact Riemann surface is a branched covering of the Riemann sphere $\mathbb{P}^{1}$. Thus, Observation 3.5 implies that the infimum in Definition 3.6 is attained.

In [2], the authors find a formula for the complexity of a Riemann surface.

Theorem 3.7 ([2, Theorem 5.4]). Let $M$ be a Riemann surface of genus $g \geq 1$. Suppose that the complexity $C_{\mathrm{Riem}}(M)$ of $M$ is finite. Let $m_{\min }$ be the minimum total length of an abstract branch triplet that is realizable on $M$. Then

$$
C_{\text {Riem }}(M)=2 \pi\left(m_{\min }+2 g-2\right) .
$$

We now find a lower bound for the number $m_{\min }$.

Proposition 3.8. The number $m_{\min }$ from equation (3.1) is greater than or equal to 3 .

Proof. Let $\mathcal{T}=(d, n, \Pi)$ be an abstract branch triplet that is realizable on $M$. We prove that the total length of $\mathcal{T}$ is always greater than or equal to three. Let $p: M \rightarrow \mathbb{P}^{1}$ be the $(d, n)$-branched covering associated to $\mathcal{T}$.

Suppose that $y_{1}, y_{2}, \ldots, y_{n}$ are all the distinct branch points of the branched covering $p$. For each $i=1,2, \ldots, n$,

- let $\Pi_{i}$ be the partition of $d$ given by the ramification indices of the points in $p^{-1}\left(y_{i}\right)$;

- let $m_{i}$ be the length of the partition $\Pi_{i}$.

Since $m=m_{1}+m_{2}+\cdots+m_{n}$ and each $m_{i} \geq 1(i=1,2, \ldots, n)$, then $m \geq n$. It remains to prove that $n \geq 3$.

Since the abstract branch triplet $\mathcal{T}$ is compatible with $M$, then

$$
m-\chi(M)=d(n-2) .
$$

Equation (3.2) implies that $n-2>0$, as $m>0, \chi(M) \leq 0$ and $d>0$.

Definition 3.9. A compact Riemann surface $M$ is called hyperelliptic if there exists a double branched covering $p: M \rightarrow \mathbb{P}^{1}$. 
Notice that our definition allows the existence of hyperelliptic Riemann surfaces of genus 0 and 1 .

Applying the Riemann-Hurwitz formula to a double branched covering we obtain the following.

Lemma 3.10. Let $p: M \rightarrow \mathbb{P}^{1}$ be a $(2, n)$-branched covering. If the genus of $M$ is equal to $g \geq 0$, then $n=2 g+2$.

Notation 3.11. Given a surface $S$, we will use the symbol $\mathbb{X}_{S}$ to denote the set of all Riemann surfaces that are homeomorphic to $S$.

The following is a well-known result (see for example [5]).

Proposition 3.12. For every surface $S$ there exists a hyperelliptic Riemann surface in $\mathbb{X}_{S}$.

We now define a notion of complexity for topological surfaces.

Definition 3.13. We define the topological complexity, $C_{\text {top }}(S)$, of a surface $S$ as the infimum of the set of all complexities of the Riemann surfaces in $\mathbb{X}_{S}$ :

$$
C_{\text {top }}(S)=\inf \left\{C_{\text {Riem }}(M) \mid M \in \mathbb{X}_{S}\right\} .
$$

Given a surface $S$, Proposition 3.12 shows that the set of all complexities of the Riemann surfaces in $\mathbb{X}_{S}$ is not empty. Thus, by Observation 3.5 there exists a Riemann surface $M \in \mathbb{X}_{S}$ such that $C_{\text {top }}(S)=C_{\text {Riem }}(M)$.

In the rest of this section we prove that the topological complexity of a surface is always finite. We start with the case of surfaces with positive genus.

Proposition 3.14. The topological complexity of a surface of genus $g \geq 1$ is finite.

Proof. Let $S$ be a surface of genus $g \geq 1$. By Proposition 3.12 there exists a hyperelliptic Riemann surface $M \in \mathbb{X}_{S}$. Let $p: M \rightarrow \mathbb{P}^{1}$ be the associated $(2, n)$ branched covering. By Lemma 3.10, $n=2 g+2$. Since $g \geq 1$, then $n=2 g+2 \geq 4$, and so $C_{\mathrm{Cov}}(p)<\infty$. Thus,

$$
C_{\text {top }}(S) \leq C_{\text {Riem }}(M) \leq C_{\text {Cov }}(p)<\infty
$$

The above proof does not work for $g=0$, because, in that case, $C_{\mathrm{Cov}}(p)=\infty$. However, we can complement Proposition 3.14 as follows.

Proposition 3.15. The topological complexity of the sphere is finite.

The proof of Proposition 3.15 follows from Lemma 2.7 and Corollary 3.4 The details are left to the reader.

Combining our last two results, we obtain the following.

Theorem 3.16. The topological complexity of a surface is finite. 


\section{The MAIN THEOREMS}

In this section we compute the topological complexity of all surfaces. We start with the case of surfaces with positive genus.

Theorem 4.1. Let $S$ be a surface of genus $g \geq 1$. Then the topological complexity $C_{\text {top }}(S)$ of $S$ is equal to $2 \pi(2 g+1)$.

Proof. By Theorem 3.16 $C_{\text {top }}(S)<\infty$. Let $M$ be a Riemann surface in $\mathbb{X}_{S}$ such that $C_{\text {Riem }}(M)=C_{\text {top }}(S)$. Let $m_{\text {min }}$ be the minimum total length of an abstract branch triplet that is realizable on $M$. By Theorem 3.7

$$
C_{\text {Riem }}(M)=2 \pi\left(m_{\min }+2 g-2\right) .
$$

Let $d=2 g+1$. Consider the abstract branch triplet $\mathcal{T}=(d, 3,\{[d],[d],[d]\})$ of total length $m_{\mathcal{T}}=3$. By Theorem 2.8 the abstract branch triplet $\mathcal{T}$ is realizable on a Riemann surface $M^{\prime} \in \mathbb{X}_{S}$. Let $p^{\prime}: M^{\prime} \rightarrow \mathbb{P}^{1}$ be the $(d, n)$-branched covering associated to $\mathcal{T}$. Since $n=3$, then $C_{\text {Cov }}\left(p^{\prime}\right)<\infty$, and so

$$
C_{\text {Riem }}\left(M^{\prime}\right) \leq C_{\text {Cov }}\left(p^{\prime}\right)<\infty \text {. }
$$

Let $m_{\min }^{\prime}$ be the minimum total length of an abstract branch triplet that is realizable on $M^{\prime}$. By Theorem 3.7

$$
C_{\text {Riem }}\left(M^{\prime}\right)=2 \pi\left(m_{\min }^{\prime}+2 g-2\right) \text {. }
$$

Since $C_{\text {Riem }}(M)=C_{\text {top }}(S) \leq C_{\text {Riem }}\left(M^{\prime}\right)$, then equations (4.1) and 4.2 imply that $m_{\min } \leq m_{\text {min }}^{\prime}$. Further, $m_{\text {min }}^{\prime} \leq m_{\mathcal{T}}=3$, as $\mathcal{T}$ is realizable on $M^{\prime}$. Therefore, $m_{\min } \leq 3$. On the other hand, by Proposition $3.8, m_{\min } \geq 3$. Hence, $m_{\min }=3$, and so we obtain:

$$
C_{\text {top }}(S)=C_{\text {Riem }}(M)=2 \pi(3+2 g-2)=2 \pi(2 g+1) .
$$

To compute the topological complexity of the sphere we need the following lemma.

Lemma 4.2. Let $p: M \rightarrow \mathbb{P}^{1}$ be a hyperbolic $(d, n)$-branched covering with $M$ homeomorphic to the sphere. Then:

(1) $d \geq 3$, and

(2) $C_{\mathrm{Cov}}(p) \geq 6 \pi$.

Proof. (1) It suffices to prove that if $d \leq 2$ then $n<3$. This is obvious for $d=1$ and it follows from the Riemann-Hurwitz formula for $d=2$.

(2) By (1), $d \geq 3$. Further, $n \geq 3$ and $C_{\mathrm{Cov}}(p)=2 \pi d(n-2)$. Therefore,

$$
C_{\mathrm{Cov}}(p)=2 \pi d(n-2) \geq 2 \pi(3)(3-2)=6 \pi \text {. }
$$

The following result complements Theorem 4.1 .

Theorem 4.3. The topological complexity of the sphere is equal to $6 \pi$. 
Proof. Let $\mathbb{S}^{2}$ denote the 2-sphere. Consider the abstract branch triplet $\mathcal{T}=$ $(3,3,\{[3],[1,2],[1,2]\})$. By Lemma 2.7, $\mathcal{T}$ is realizable on a Riemann surface $M_{0} \in \mathbb{X}_{\mathbb{S}^{2}}$. Let $p_{0}: M_{0} \rightarrow \mathbb{P}^{1}$ be a $(d, n)$-branched covering whose branch data is equal to $\mathcal{T}$. Since $d=3$ and $n=3$, then $C_{\mathrm{Cov}}\left(p_{0}\right)=2 \pi d(n-2)=6 \pi$. Hence,

$$
C_{\text {top }}\left(\mathbb{S}^{2}\right) \leq C_{\text {Riem }}\left(M_{0}\right) \leq C_{\text {Cov }}\left(p_{0}\right)=6 \pi \text {. }
$$

Let $M \in \mathbb{X}_{\mathbb{S}^{2}}$ and suppose that $p: M \rightarrow \mathbb{P}^{1}$ is a $(d, n)$-branched covering. If $p$ is hyperbolic, then Lemma 4.2 implies that $C_{\mathrm{Cov}}(p) \geq 6 \pi$. If $p$ is not hyperbolic, $C_{\text {Cov }}(p)=\infty$. In either case, $C_{\text {Cov }}(p) \geq 6 \pi$. Thus, $C_{\text {Riem }}(M) \geq 6 \pi$.

Since $C_{\text {Riem }}(M) \geq 6 \pi$ for all $M \in \mathbb{X}_{\mathbb{S}^{2}}$, then $C_{\text {top }}\left(\mathbb{S}^{2}\right) \geq 6 \pi$. Combining this with equation 4.3 , we obtain that $C_{\text {top }}\left(\mathbb{S}^{2}\right)=6 \pi$.

We can merge Theorems 4.1 and 4.3 into the following result.

Theorem 4.4. Let $C_{\text {top }}(S)$ denote the topological complexity of a surface $S$ of genus $g$. Then

$$
C_{\mathrm{top}}(S)= \begin{cases}2 \pi(2 g+1) & \text { if } g \geq 1, \\ 6 \pi & \text { if } g=0\end{cases}
$$

\section{The Simple COMPleXity OF A TOPOLOGICAL SURFACE}

In this section we define a special type of $(d, n)$-branched coverings that we call simple. As usual, $|B|$ denotes the cardinality of the set $B$.

Definition 5.1. A $(d, n)$-branched covering $p: M \rightarrow \mathbb{P}^{1}$ is called simple if $\left|p^{-1}(y)\right| \geq$ $d-1$ for all $y \in \mathbb{P}^{1}$.

For simple branched coverings, the Riemann-Hurwitz formula takes the following form.

Theorem 5.2. Let $p: M \rightarrow S^{2}$ be a simple $(d, n)$-branched covering. Let $g$ be the genus of $M$. Then

$$
2-2 g=2 d-n .
$$

We use Theorem 5.2 to obtain a lower bound for the complexity of a simple branched covering.

Lemma 5.3. Let $p: M \rightarrow \mathbb{P}^{1}$ be a simple $(d, n)$-branched covering. Suppose that the genus $g$ of $M$ is positive. Then $C_{\mathrm{Cov}}(p) \geq 8 \pi g$.

Proof. If $n<3$ the conclusion of the lemma is clearly satisfied. We assume that $n \geq 3$ for the rest of the proof, which implies that $d \geq 2$.

By Theorem $5.2 n=2 d+2 g-2 \geq 4$. Therefore,

$$
C_{\mathrm{Cov}}(p)=2 \pi d(n-2)=2 \pi d(2 d+2 g-4)=4 \pi d(d+g-2) \geq 4 \pi(2)(2+g-2)=8 \pi g .
$$

We now define a notion of simple complexity for topological surfaces. 
Definition 5.4. We define the simple topological complexity, $C_{\text {simp }}(S)$, of a surface $S$ as the infimum of the set of all complexities of simple branched coverings of $M$ to $\mathbb{P}^{1}$ with $M \in \mathbb{X}_{S}$ :

$C_{\text {simp }}(S)=\inf \left\{C_{\text {Cov }}(p) \mid p: M \rightarrow \mathbb{P}^{1}\right.$ is a simple branched covering and $\left.M \in \mathbb{X}_{S}\right\}$.

Proposition 3.12 and Observation 3.5 imply that the infimum in Definition 5.4 is attained, although it could be infinite.

We now compute the simple topological complexity of surfaces of positive genus.

Theorem 5.5. Let $S$ be a surface of genus $g \geq 1$. Then the simple topological complexity $C_{\text {simp }}(S)$ of $S$ is equal to $8 \pi g$.

Proof. By Proposition 3.12 there exists a hyperelliptic Riemann surface $M_{0} \in \mathbb{X}_{S}$. Let $p_{0}: M_{0} \rightarrow \mathbb{P}^{1}$ be the associated $(2, n)$-branched covering. By Lemma 3.10 $n=2 g+2 \geq 4$. Therefore,

$$
C_{\mathrm{Cov}}\left(p_{0}\right)=2 \pi d(n-2)=2 \pi(2)(2 g)=8 \pi g .
$$

Since $p_{0}$ is simple, then $C_{\text {simp }}(S) \leq C_{\text {Cov }}\left(p_{0}\right)=8 \pi g$. By Lemma $5.3, C_{\text {simp }}(S) \geq$ $8 \pi g$. Combining the last two inequalities, we obtain that $C_{\text {simp }}(S)=8 \pi g$.

Before extending Theorem 5.5 to the case $g=0$, we need the following.

Lemma 5.6. Let $p: M \rightarrow \mathbb{P}^{1}$ be a simple $(d, n)$-branched covering with $M \in \mathbb{X}_{\mathbb{S}^{2}}$. Then $C_{\mathrm{Cov}}(p) \geq 12 \pi$.

The proof of Lemma 5.6 is similar to that of Lemma 5.3 and left to the reader.

We state the analog of Theorem 5.5 for genus zero.

Theorem 5.7. The simple topological complexity of the sphere is equal to $12 \pi$.

Proof. Consider the abstract branch triplet $\mathcal{T}=(3,4,\{[1,2],[1,2],[1,2],[1,2]\})$. By Theorem 2.9 , there exists a $(3,4)$-branched covering $p_{0}: M_{0} \rightarrow \mathbb{P}^{1}$, with $M_{0} \in \mathbb{X}_{\mathbb{S}^{2}}$, whose branch triplet coincides with $\mathcal{T}$. This branched covering is simple because the length of the partition $[1,2]$ is equal to $2=d-1$. Further,

$$
C_{\text {simp }}\left(\mathbb{S}^{2}\right) \leq C_{\text {Cov }}\left(p_{0}\right)=2 \pi d(n-2)=2 \pi(3)(4-2)=12 \pi .
$$

By Lemma 5.6 $C_{\text {simp }}\left(\mathbb{S}^{2}\right) \geq 12 \pi$. Combining the last two inequalities, we obtain that $C_{\text {simp }}\left(\mathbb{S}^{2}\right)=12 \pi$.

We can merge Theorems 5.5 and 5.7 into the following result.

Theorem 5.8. Let $C_{\text {simp }}(S)$ denote the simple topological complexity of a surface $S$ of genus $g$. Then

$$
C_{\text {simp }}(S)= \begin{cases}8 \pi g & \text { if } g \geq 1 \\ 12 \pi & \text { if } g=0\end{cases}
$$




\title{
REFERENCES
}

[1] Krzysztof Barański. On realizability of branched coverings of the sphere. Topology Appl. 116 (2001), no. 3, 279-291. MR 1857667.

[2] Aldo-Hilario Cruz-Cota and Teresita Ramirez-Rosas. The complexity of Riemann surfaces and the Hurwitz existence problem. Bull. Aust. Math. Soc. 87 (2013), no. 1, 131-138. MR 3011948

[3] Allan L. Edmonds, Ravi S. Kulkarni, and Robert E. Stong. Realizability of branched coverings of surfaces. Trans. Amer. Math. Soc. 282 (1984), no. 2, 773-790. MR 0732119

[4] Cloyd L. Ezell. Branch point structure of covering maps onto nonorientable surfaces. Trans. Amer. Math. Soc. 243 (1978), 123-133. MR 0500900

[5] Otto Forster. Lectures on Riemann surfaces, volume 81 of Graduate Texts in Mathematics. Springer-Verlag, New York, 1991. MR 1185074

[6] S. M. Gersten. On branched covers of the 2-sphere by the 2-sphere. Proc. Amer. Math. Soc. 101 (1987), no. 4, 761-766. MR 0911047.

[7] A. Hurwitz. Ueber Riemann'sche Flächen mit gegebenen Verzweigungspunkten. Math. Ann. 39 (1891), no. 1, 1-60. MR 1510692.

[8] Dale H. Husemoller. Ramified coverings of Riemann surfaces. Duke Math. J. 29 (1962), 167174. MR 0136726.

[9] F. Pakovich. Solution of the Hurwitz problem for Laurent polynomials. J. Knot Theory Ramifications 18 (2009), no. 2, 271-302. MR 2507927

[10] Ekaterina Pervova and Carlo Petronio. On the existence of branched coverings between surfaces with prescribed branch data. I. Algebr. Geom. Topol. 6 (2006), 1957-1985. MR 2263056.

[11] Ekaterina Pervova and Carlo Petronio. On the existence of branched coverings between surfaces with prescribed branch data. II. J. Knot Theory Ramifications 17 (2008), no. 7, 787-816. MR 2436584

\author{
A.-H. Cruz-Cota \\ Texas Wesleyan University, Fort Worth, TX, USA \\ acruz-cota@txwes.edu
}

Received: April 25, 2017

Accepted: April 17, 2018 